\title{
A portrait of 4979 Otawara, target of the Rosetta space mission ${ }^{\star}$
}

\author{
S. Fornasier ${ }^{1,2}$, M. A. Barucci ${ }^{2}$, R. P. Binzel ${ }^{3}$, M. Birlan ${ }^{2}$, M. Fulchignoni ${ }^{2}$, C. Barbieri ${ }^{1}$, S. J. Bus ${ }^{4}$, A. W. Harris ${ }^{5}$, \\ A. S. Rivkin ${ }^{3}$, M. Lazzarin ${ }^{1}$, E. Dotto ${ }^{6,7}$, T. Michałowski ${ }^{8}$, A. Doressoundiram ${ }^{2}$, \\ I. Bertini ${ }^{1}$, and N. Peixinho ${ }^{2,9}$
}

1 Astronomical Department of Padova, Vicolo dell'Osservatorio 2, 35122 Padova, Italy e-mail: [fornasier; barbieri; lazzarin]@pd.astro.it, bertini@planet.pd.astro.it

2 LESIA, Observatoire de Paris, 92195 Meudon Principal Cedex, France e-mail: [antonella.barucci; Mirel.Birlan; Marcello.Fulchignoni; alain.doressoundiram; Nuno.Peixinho]@obspm.fr

3 Department of Earth, Atmospheric and Planetary Sciences, Massachusetts Institute of Technology, Cambridge, 02139, USA e-mail: [rpb; asrivkin]@mit.edu

4 Institute for Astronomy Hilo, HI 96720, USA e-mail: sjb@ifa.hawaii.edu

5 Space Science Institute, 4603 Orange Knoll Ave., La Canada, CA 91011-3364, USA e-mail: harrisaw@colorado.edu

${ }^{6}$ INAF-Osservatorio Astronomico di Torino, Strada Osservatorio 20, 10025 Pino Torinese (TO), Italy e-mail: dotto@to.astro.it

7 INAF-Osservatorio Astronomico di Roma, Via Frascati 33, 00040, Monte Porzio Catone (Roma), Italy

8 Astronomical Observatory, Adam Mickiewicz University, Sloneczna 36, 60-286 Poznan, Poland e-mail: tmich@amu.edu.pl

9 CAAUL, Tapada da Ajuda, 1349-018 Lisboa, Portugal

Received 27 May 2002 / Accepted 17 October 2002

\begin{abstract}
A physical portrait based on spectral and photometric data of 4979 Otawara, the first asteroid target of the Rosetta mission, is presented. The aim of this work is to investigate the composition of 4979 Otawara and to evaluate its rotation pole orientation. The spectroscopic observations obtained at the Palomar 200" and IRTF telescopes cover the wavelength range 0.4 to $2.5 \mu \mathrm{m}$, and provide a definitive classification of Otawara as an S-type asteroid. An analysis of band depths and slopes places Otawara in the S(IV) subgroup, suggesting a similarity to ordinary chondrite meteorites.

Moreover we present new photometric data, obtained at the Asiago Observatory and at the TNG telescope, that allow confirmation of the fast rotational period of $2.707 \pm 0.005$ hours, and a first indication of the spin vector of Otawara.
\end{abstract}

Key words. planets and satelites: individual: Otawara - minor planets, asteroids

\section{Introduction}

Rosetta is the cornerstone mission of ESA devoted to the study of minor bodies of the Solar System. The mission will be launched in January 2003 and has as its primary target 46P/Wirtanen, a short period comet in the Jupiter family whose

Send offprint requests to: S. Fornasier,

e-mail: fornasier@pd.astro.it

^ Based on observations obtained at the IRTF Observatory, Hawaii, USA, at the Palomar Mountain Observatory, California, USA, at the TNG telescope, La Palma, Spain and at the Asiago Astrophysical Observatory, Asiago, Italy. rendezvous will be in November 2011. During its cruise to comet Wirtanen, the Rosetta spacecraft will encounter two main belt asteroids: 4979 Otawara and 140 Siwa. Rosetta will fly by Otawara on 11 July 2006, at a heliocentric distance of 1.89 AU, with a minimum encounter distance of $2200 \mathrm{~km}$ and a relative velocity of about $10 \mathrm{~km} \mathrm{~s}^{-1}$. The Siwa flyby will occur on 24 July 2008 at 2.75 AU from the Sun, at a minimum distance of $3500 \mathrm{~km}$. The goals of the mission during the asteroid encounters will be a complete determination of their physical properties (size, shape, density, mass, rotational properties), the study of their composition, and the investigation of the neighbouring space in order to detect possible satellites. 
Table 1. Orbital and physical characteristics of 4979 Otawara. ${ }^{a}$ data from Doressoundiram et al. (1999).

\begin{tabular}{ll}
\hline \hline perihelion distance (AU) & 1.855 \\
aphelion (AU) & 2.481 \\
semimajor axis (AU) & 2.169 \\
eccentricity & 0.144 \\
inclination (degrees) & 0.912 \\
synodical rotation period (hrs) & $2.707 \pm 0.005^{a}$ \\
taxonomy & ${\mathrm{S} \mathrm{or} \mathrm{V}^{a}}^{a}$ \\
absolute magnitude & $14.08 \pm 0.04^{a}(\mathrm{~S}-$ type $)$ \\
circular effective radius & $2.0 \mathrm{~km}^{a}(\mathrm{~S}-$ type $)$ \\
density & $\geq 1.9^{a}$ \\
\hline
\end{tabular}

Otawara is a small main-belt asteroid. At the time of Rosetta asteroid target definition, very little was known about the physical properties of this object. Successive international campaigns have revealed that Otawara is a very interesting target; it has a rotational period of only 2 hours and $42 \mathrm{~min}$ (Doressoundiram et al. 1999). It will be the first fast rotating asteroid encountered by a space mission. The fast rotation of Otawara will enable Rosetta to image and measure the asteroid's characteristics during one complete rotation.

In this paper we present the first near infrared spectrum of Otawara that definitively determines its taxonomic class. We add also a new visible spectrum of the object and new photometric data that confirm the rotational period value previously determined by Doressoundiram et al. (1999), and Le Bras et al. (2001) and allow a first estimation of the spin vector state.

This information will aid Rosetta mission planners in optimising the encounter trajectory and planning of science operations.

\section{Observations and data reduction}

\subsection{Photometric measurements}

Photometric observations of Otawara were carried out at the Asiago Astrophysical Observatory, Italy, and at the Telescopio Nazionale Galileo (TNG), La Palma, Spain. Unfortunatly, 2 nights devoted to Otawara observations at the Asiago telescope were lost due to bad weather conditions, and we could observe only partially during two other nights (one at the Asiago telescope and one at the TNG telescope) in poor photometric conditions. So for both nights only relative photometry between the asteroid and some field comparison stars was possible.

At Asiago we observed on 23 December 2001 using the $1.82 \mathrm{~m}$ telescope equipped with the AFOSC camera and a $1024 \times 1024$ pixel CCD, with a total field of view of $8.14 \times$ $8.14 \mathrm{arcmin}$. The pixel size is $24 \mu \mathrm{m}$ and the pixel scale is $0.473 \mathrm{arcsec} / \mathrm{px}$. Our measurements were made with a $V$ filter (Johnson) and 300-420 s of exposure time. At the TNG $3.5 \mathrm{~m}$ telescope we observed on 8 January 2002, with the OIG camera equipped with a mosaic of two thinned and back-illuminated EEV 42-80 CCDs with $2048 \times 4096$ pixels each (pixel size of 13.5 microns). The resulting pixel scale is $0.072 \mathrm{arcsec} / \mathrm{pix}$ for a total field of view of about $4.9 \times 4.9$ arcmin. We observed both in $V$ and $R$ filters (Johnson) and the exposure times ranged from 60 to $360 \mathrm{~s}$.

Data reduction was performed in the following way: images were bias subtracted and divided by a flat field (computed as a median of several flat fields obtained during twilight), then relative magnitudes were computed using aperture photometry. We used an aperture radius of about twice the average seeing, and sky subtraction was performed using a 5-10 pixels wide annulus around the asteroid or reference stars.

\subsection{Visible spectrum}

The visible spectrum of Otawara was obtained at Palomar Mountain Observatory on 23 December 2001 with the 200-inch (5-m) telescope equipped with the Double Spectrograph (see Fig. 2). The instrument simultaneously obtains the blue and red halves of a long slit spectrum using a pair of CCD cameras. Different focal lengths of the two cameras give a pixel scale of 1.25 and 0.94 arcsec per pixel (the $2 \times 2$ binned mode was used) respectively for the blue and red channels. A 300 line/mm grating was used for the blue channel, covering a spectral range of 0.42 to $0.62 \mu \mathrm{m}$ and giving a dispersion of $7 \AA$ per binned pixel. A 158 line $/ \mathrm{mm}$ grating was used for the red part, covering the wavelength range from 0.53 to $0.92 \mu \mathrm{m}$ with a dispersion of $10 \AA$ per binned pixel. The final spectrum is the mean of three blue/red pair exposures of $600 \mathrm{~s}$ each. A slit of 6 arcsec was used to acquire the data, oriented in a $\mathrm{N}-\mathrm{S}$ direction in order to minimize effects due to differential atmospheric refraction for objects observed near the meridian. Solar analog stars were observed at airmasses close to that of Otawara.

Data were reduced following usual reduction procedures for visible spectroscopy (see Bus 1999; Xu et al. 1995). Upon extraction each spectrum was rebinned to a uniform dispersion of $25 \AA / p x$ and the solar contribution was removed by division by a solar analog star. All the spectra were normalized at $5500 \AA$ A.

\subsection{Infrared spectrum}

The infrared spectrum of Otawara was obtained at the NASA Infrared Telescope Facility (IRTF), Hawaii, USA, equipped with the medium-resolution infrared spectrograph SpeX and employing a $1024 \times 1024 \mathrm{InSb}$ array (Rayner et al. 1998). Each exposure consisted of 32 non-destructive reads providing a readout noise of about $15 \mathrm{e}^{-}$RMS. Otawara was observed on 12 January 2002 in remote mode from the Observatoire de Paris-Meudon. The instrument was used in the low-resolution prism mode that allows a single exposure to cover the wavelength range from 0.8 to $2.5 \mu \mathrm{m}$, with a dispersion of about $50 \AA$ per pixel. The observations were made using a slit of 0.8 arcsec (oriented in the $\mathrm{N}-\mathrm{S}$ direction) nodding the object along the spatial direction of the slit (offset of $7.5 \mathrm{arcsec}$ ), in order to obtain alternated pairs of exposures (denoted as A and B). This procedure allows a very close sky and bias measurement in the same pixel positions as the spectral measurement. A sequence of 9 cycles, each one of the type ABBA, with four exposures of 
Table 2. 4979 Otawara: observational circumstances, where $r$ and $\Delta$ are respectively the heliocentric and geocentric distances of the asteroid, $\alpha$ the phase angle of the asteroid at the time of observations and $m_{v}$ is the predicted magnitude from the JPL ephemeris service. The date indicated is relative to the beginning of observations; the symbol * means that observations ended the following day.

\begin{tabular}{|c|c|c|c|c|c|c|c|c|c|}
\hline Date & $\begin{array}{l}\text { UT } \\
\text { start }\end{array}$ & $\begin{array}{l}\text { UT } \\
\text { end }\end{array}$ & Telescope & Mode & Instrument & $\overline{m_{v}}$ & $r(\mathrm{AU})$ & $\overline{\Delta(\mathrm{AU})}$ & $\bar{\alpha}$ \\
\hline 23 Dec. 01 & 08:54 & 09:18 & Palomar 200" & Vis. spectrum & Double Spec. & 17.27 & 2.480981 & 1.512879 & $5: 16$ \\
\hline 23 Dec. 01 & $21: 48$ & $01: 43 *$ & Asiago $1.8 \mathrm{~m}$ & $V$ imaging & AFOSC & 17.26 & 2.480928 & 1.511215 & 4.87 \\
\hline 08 Jan. 02 & $21: 47$ & $01: 25^{*}$ & TNG $3.5 \mathrm{~m}$ & $V+R$ imaging & OIG & 17.15 & 2.478565 & 1.501553 & 3.32 \\
\hline 12 Jan. 02 & 09:55 & $11: 21$ & IRTF $3.0 \mathrm{~m}$ & IR spectrum & SpeX & 17.26 & 2.477813 & 1.509438 & 5.09 \\
\hline
\end{tabular}

2 min each, was obtained for a total integration time of $72 \mathrm{~min}$. The object was observed on the meridian, with an airmass less than 1.1 , in order to minimize effects due to differential refraction. A solar analog star, Hyades 64, was observed just before and after (and at very similar airmasses) as the object and was used to normalize the reflectance spectrum of the asteroid. Flat field and arcline spectra were also acquired for data calibration.

Data reduction was performed in the traditional way for IR observations: first spectra were corrected for flat fielding, then bias and sky subtraction was performed by producing A-B and B-A frames. The next step involved shift and add of the positive spectrum of $(B-A)$ frame on the positive spectrum of A-B frame. The final spectrum (Fig. 2) is the result of the mean of all pairs of frames previously combined. The spectrum was then extracted and wavelength calibrated using the arcline spectrum of an Argon lamp. Finally, the extinction correction and solar removal was obtained by division of the asteroid spectrum with that of the solar analog star Hyades 64 .

\section{Analysis}

\subsection{Lightcurve and spin state}

The synodic rotational period was determined by applying a Fourier analysis to our photometric data, as described by Harris et al. (1989). The value of the synodic rotational period that best fits our observations is $P_{\text {syn }}=2.707 \pm 0.005$ hours. This result was already found by Doressoundiram et al. (1999) and confirmed by Le Bras et al. (2001). Figure 1 shows the composite lightcurve obtained by combining in rotational phase all of our photometric data.

Since no absolute calibration was obtained, due to the nonphotometric sky conditions, an offset was applied to each night of data, in order to center the lightcurve on the mean brightness of the asteroid. $R$-filter and $V$-filter composite lightcurves, which show similar behaviour, have been superimposed. The obtained amplitude is $0.22 \pm 0.03 \mathrm{mag}$.

The spin vector, sidereal period, and triaxial ellipsoid model for a given asteroid can be determined using the method described by Michałowski (1993). In this method the epochs of brightness maxima, amplitudes and magnitudes are considered. The results are obtained by building a set of nonlinear equations whose solution is found by the least square fitting.

We have been able to use for Otawara the epochs, amplitudes and $R$ magnitudes from December 1998-January 1999 (Doressoundiram et al. 1999) and July-August 2000

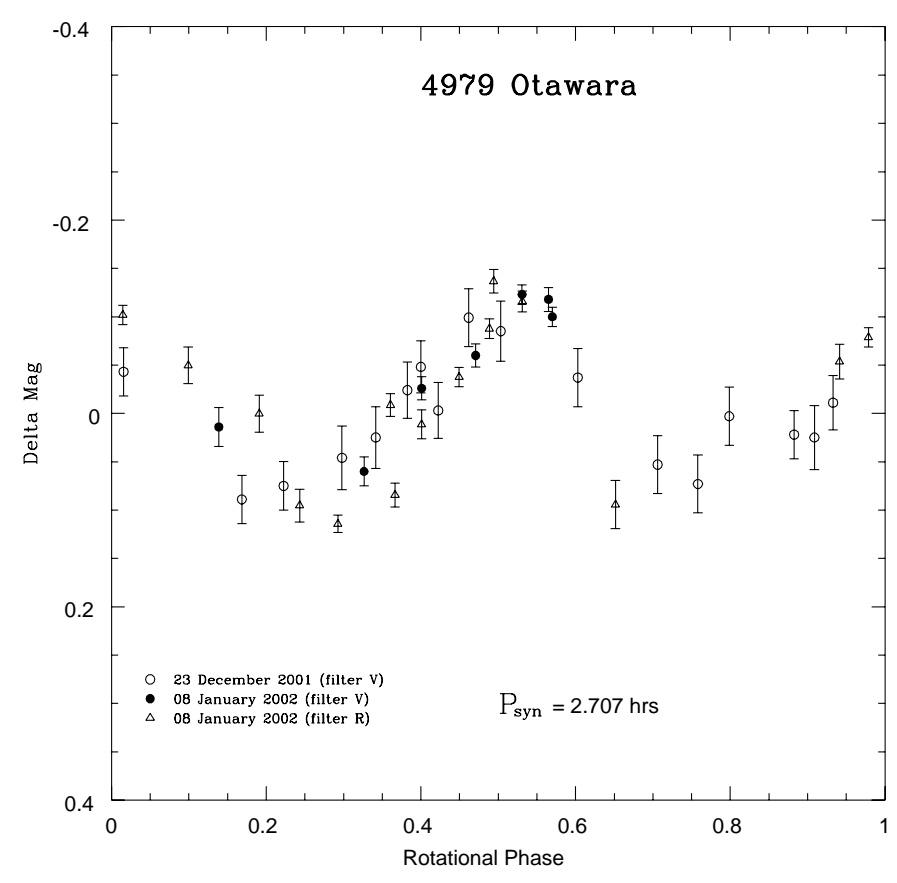

Fig. 1. Composite lightcurve of 4979 Otawara. The error bars contain the photon noise. The zero phase is at JD 2452276.5.

(Le Bras et al. 2001) and only the epochs and amplitudes obtained in December 2001-January 2002 (this paper). The ecliptic longitude $(\lambda)$ and latitude $(\beta)$ of Otawara during these observations are the following:

$\begin{array}{ccc}\text { Date } & \lambda & \beta \\ \text { 23 Jan. 1999 } & 123.5^{\circ} & +1.0^{\circ} \\ \text { 06 Aug. 2000 } & 314.3^{\circ} & -1.3^{\circ} \\ \text { 01 Jan. 2002 } & 101.9^{\circ} & +0.6^{\circ}\end{array}$

It should be noted that these observations were obtained from rather similar geometric configurations of Otawara as the difference in ecliptic longitudes between the 1998/99 and 2001/02 oppositions was smaller than $30^{\circ}$ and the longitude difference was close to $180^{\circ}$ between 2000 and 1998/99 apparitions. For that reason, the available observational data are far from ideal as required for the method mentioned above. The observed amplitudes of Otawara have been reduced to zero phase angle with the amplitude-phase relationship described by Zappala et al. (1990). The correction coefficient was found to be $m=0.006$. The $H G$-magnitude system (Bowell et al. 1989) has allowed us to reduce the $R$ magnitudes to zero phase angle and we have obtained $G_{R}=0.19$. 
Despite the limitation in the observational data we have obtained some preliminary results for Otawara. The sense of rotation seems to be retrograde. The sidereal period $\left(P_{\text {sid }}\right)$, the ecliptic coordinates of the asteroid north pole $\left(\lambda_{\mathrm{p}}, \beta_{\mathrm{p}}\right)$, the $a / b$ ratio of the triaxial ellipsoid shape, the absolute $R$ magnitude for the aspect $90^{\circ} H_{R}(90,0)$, and their formal errors are as follows:

$$
\begin{aligned}
& P_{\text {sid }}=0.112776 \pm 0.000001 \text { (days) } \\
& \lambda_{\mathrm{p}}=50^{\circ} \pm 5^{\circ} \\
& \beta_{\mathrm{p}}=-30^{\circ} \pm 16^{\circ} \\
& a / b=1.21 \pm 0.05 \\
& H_{R}(90,0)=13.99 \pm 0.05(\mathrm{mag}) .
\end{aligned}
$$

A second solution with the same parameters and $\lambda_{\mathrm{p}}=230^{\circ}$ has also been obtained.

According to these results, Otawara was observed close to equatorial aspects $\left(\approx 90^{\circ}\right)$. Careful examination of the relation between aspect, pole and asteroid ecliptic coordinates (Eq. (4) in Michałowski 1993) shows that equatorial aspects do not practically depend on pole latitude when the asteroid latitude is close to $0^{\circ}$, as it is for Otawara. This is probably the reason for the large formal error in $\beta_{\mathrm{p}}$.

A spurious value of the $b / c$ ratio $(2.3 \pm 0.9)$ of the triaxial ellipsoid shape comes out of the formal solution, but in this case it does not diminish the validity of the rest of the solution. In fact, the amplitude-aspect and magnitude-aspect relationships (Eqs. (3) and (5) in Michałowski 1993, respectively) show that observed amplitudes and magnitudes do not depend on $b / c$ for aspects close to equatorial ones. Therefore, we can assume that the value for $b / c$ and its formal error are solution artifacts and not real. Furthermore, because of the rapid spin rate, if the polar flattening $b / c$ is significantly greater than 1.0 , a state of tensile stress would be implied. This is highly unlikely for an asteroid of this size, thus we expect $b / c$ is not greater than about 1.2.

In order to obtain the full model for Otawara we need further observations carried out for aspects far from equatorial ones. The best observations will be during the oppositions when the ecliptic longitudes of Otawara are in the range $0^{\circ}-70^{\circ}$ or $180^{\circ}-250^{\circ}$.

The next oppositions of Otawara will be in June 2003, with an ecliptic longitude $(\lambda)$ of $263^{\circ}$, in December 2004 with $\lambda=$ $81^{\circ}$, in April 2006 with $\lambda=216^{\circ}$, in November 2007 with $\lambda=58^{\circ}$, in March 2009 with $\lambda=180^{\circ}$. So the oppositions from 2006 to 2009 fall in the desirable ecliptic longitude range.

Further ground observations are needed to improve the spin vector of Otawara, even if only the opposition in April 2006 seems to have an orbital configuration suitable for a better pole determination before the spacecraft flyby in July 2006.

\subsection{Spectral analysis}

In Fig. 2 the combined visible and infrared spectra of Otawara are shown. The visible data are normalized at $5500 \AA$ and the overlap between visible and infrared spectra was performed by

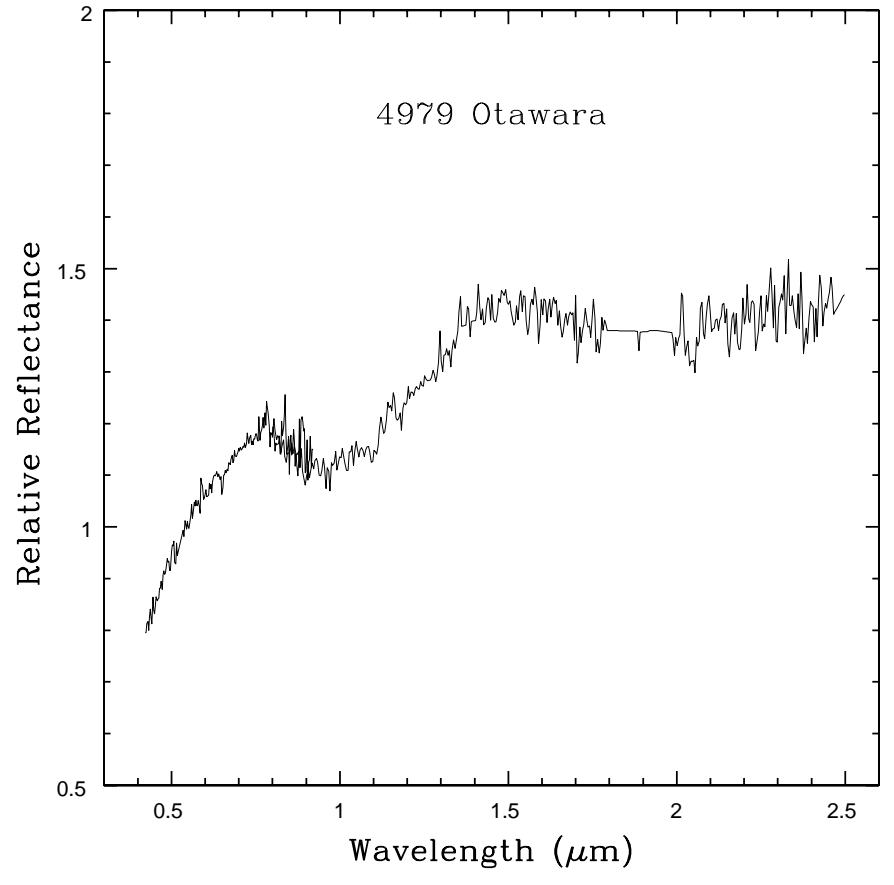

Fig. 2. Visible and infrared spectral reflectance of 4979 Otawara. The noise introduced by water band at $1.8 \mu \mathrm{m}$ has beed excluded. The spectrum is normalized at $5500 \AA$.

shifting the infrared data with a scaling factor derived by minimizing the chi-squared value of polynomial fitting to both visible and IR data. A first analysis of the spectrum allows us to determine that Otawara is an S-type asteroid. Based on only the visible spectrophotometric data, Doressoundiram et al. (1999) determined that their spectrum could be consistent with either a S- or V-type asteroid. From our IR data we can exclude any link between Otawara and V class due to the very small 2-micron absorption compared to that of a typical $\mathrm{V}$ asteroid. The shape of the spectrum over the wavelength interval $0.42-2.50 \mu \mathrm{m}$ is consistent with an S-type object.

We also compared our visible data obtained from Palomar with the visible spectrum published by Doressoundiram et al. (1999). Even though the previously published spectrum is noisier than our data, the spectral behaviour is exactly the same, confirming the visible trend previously observed.

In order to investigate possible variations of the surface properties during the rotational period, we examined the IRTF near infrared data time over 3 intervals (named I, II, III), each of them with $24 \mathrm{~min}$ of exposure time. The total elapsed time is $86 \mathrm{~min}$, corresponding to $53 \%$ of the rotational period of the asteroid. The central universal time of each of these interval is $10: 08,10: 36$ and $11: 06$, corresponding to a rotational phase of $0.40,0.57$ and 0.76 respectively. These spectra were filtered with a median filter (box size of 39 points) since they were noisier than the spectrum obtained with the total exposure.

There is no obvious difference between the three composite spectra shown in Fig. 3, meaning that for almost half of the rotational period Otawara's composition is homogeneous within $\pm 5-10 \%$ variation, the limit of our precision. 


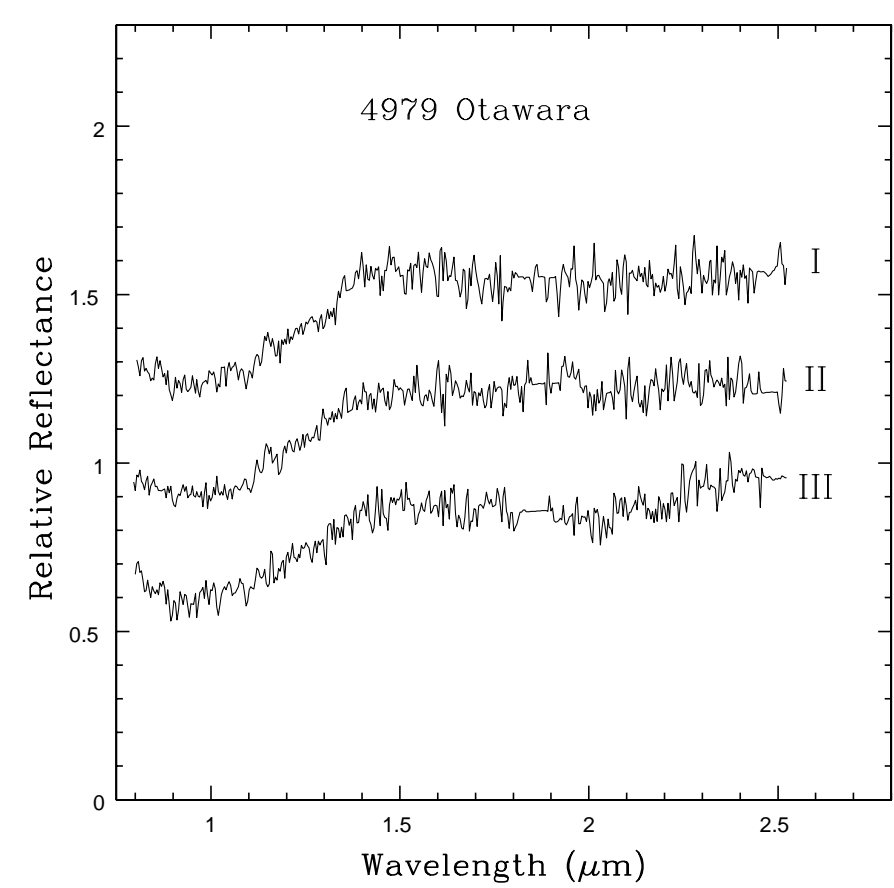

Fig. 3. Infrared spectra of 4979 Otawara: I is the spectrum relative to the first $24 \mathrm{~min}$ of integration, II that relative to the following $24 \mathrm{~min}$ and III that relative to the last $24 \mathrm{~min}$ (the total exposure time was $72 \mathrm{~min}$ ). Spectral data have been normalized at $1.25 \mu \mathrm{m}$; spectra I and III are vertically shifted by 0.3 for clarity.

We tried to better analyze the data in order to identify which S subclass of the Gaffey et al. (1993) classification scheme best describes the Otawara spectrum. In this classification, the $\mathrm{S}$ class is divided into 7 subclasses on the basis of the area ratio between the $2 \mu \mathrm{m} / 1 \mu \mathrm{m}$ band, the center position of these two bands, the depth and slope of the $1 \mu \mathrm{m}$ absorption, as these parameters are intrinsically related to the mineralogy of the asteroid surface.

The analysis of the spectrum (see the procedure described by Gaffey et al. 1993) gives the center position of the band BI (that around 1 micron) at $0.9570 \pm 0.0050 \mu \mathrm{m}$, with a band depth of $0.18 \pm 0.01$ and a spectral slope of $0.0804 \%$ per $1000 \AA$ (evaluated from $0.75 \mu \mathrm{m}$ to $1.45 \mu \mathrm{m}$ ). This band is due to the presence of both olivine and pyroxene. Regarding the band around $2 \mu \mathrm{m}$, associated with the presence of pyroxene, it is centered at $1.9780 \pm 0.0050 \mu \mathrm{m}$ and has a band depth of $0.07 \pm 0.01$.

The $2 \mu \mathrm{m} / 1 \mu \mathrm{m}$ band area ratio is $0.47 \pm 0.09$. On the basis of these values 4979 Otawara is well placed in the space of S(IV) class (see Fig. 4).

\section{Comparison to ordinary chondrites}

The assignment of Otawara to the S(IV) subclass is particularly interesting as the S(IV) asteroids are characterized by a composition that includes the silicate components of the ordinary chondrites. S(IV) members are the most probable candidates for parent bodies of ordinary chondrites among the S-type asteroid population (Gaffey et al. 1993; Gaffey 2000, 2001). For this reason, we want to investigate the possible links with OC meteorites.

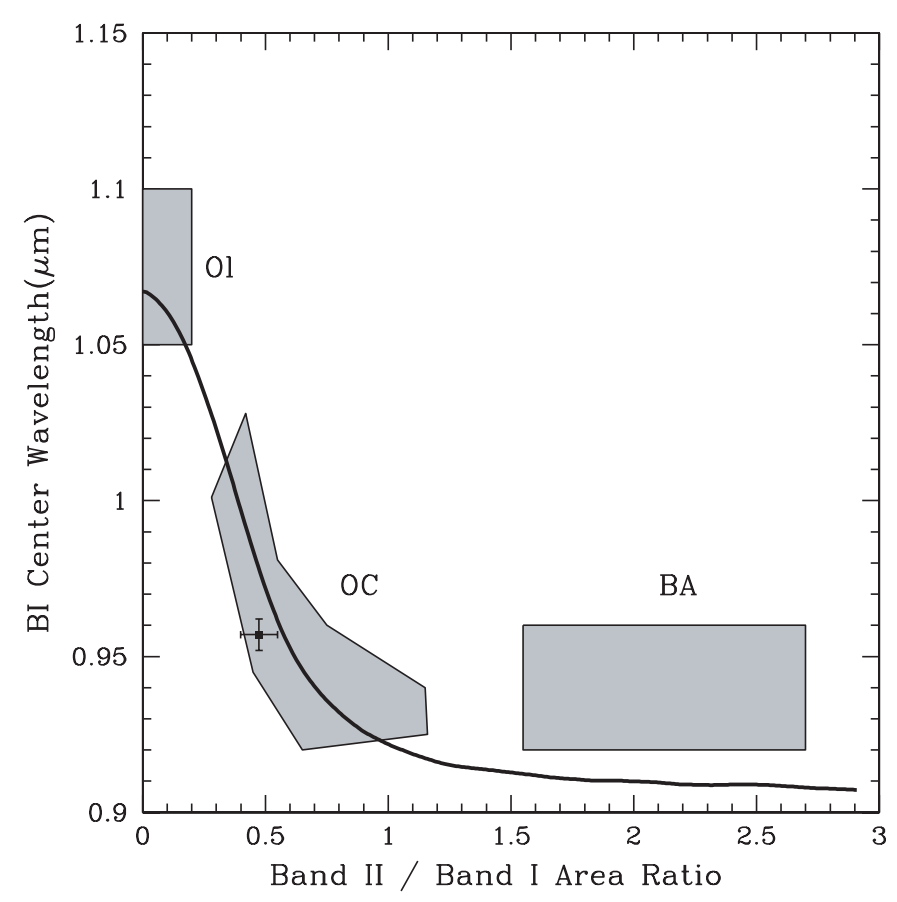

Fig. 4. BI center versus the Band II/Band I area ratio for 4979 Otawara (squared point). The three background regions are defined by meteorite spectra by Gaffey et al. (1993). The Ol rectangular region includes essentially monomineralic olivine assemblages; the OC polygonal region represents the mafic silicate components of ordinary chondrites and corresponds to the location of the S(IV) subtype in the Gaffey et al. classification scheme (1993); the BA rectangular zone includes the pyroxene dominated basaltic achondrites assemblages. The heavy solid line indicates the location of the olivine-orthopyroxene mixing line (Cloutis et al. 1986).

Plotting the BI band center position versus Band II/Band I area ratio places Otawara in the ordinary chondrite (OC) field (Fig. 4), lying near the olivine-pyroxene mixing line. This similarity to OC meteorites implies that Otawara is not a differentiated asteroid.

We analyzed the percentage of orthopyroxene present on Otawara, using the method suggested by Cloutis et al. (1986):

$\mathrm{OPX}(\%)=\frac{\mathrm{OPX}}{\mathrm{OPX}+\mathrm{OL}}=0.4187 \times\left(\frac{\mathrm{BII}}{\mathrm{BI}}+0.125\right)$

where OPX is the orthopyroxene mass fraction, OL the olivine mass fraction and BI and BII are the band area corresponding to the 1 and 2 micron absorptions, respectively.

This method was recently tested by Gaffey (1999) and Berthoud et al. (2001) on samples of ordinary chondrites, finding good agreement between the orthopyroxene abundance computed and the normative abundances derived from chemical analysis (Jarosewich 1990; McSween et al. 1991). For Otawara we find a percentage of $25 \pm 3$ of orthopyroxene, consistent with the abundance typical of L and LL ordinary chondrites.

In fact $\mathrm{S}(\mathrm{IV})$ objects are composed of olivineorthopyroxene (Ca-poor) mixtures which could be representative assemblages that are similar to undifferentiated ordinary chondrites but also to unmelted silicate portions of primitive achondrite. As the band depth at $2 \mu \mathrm{m}$ of measured 


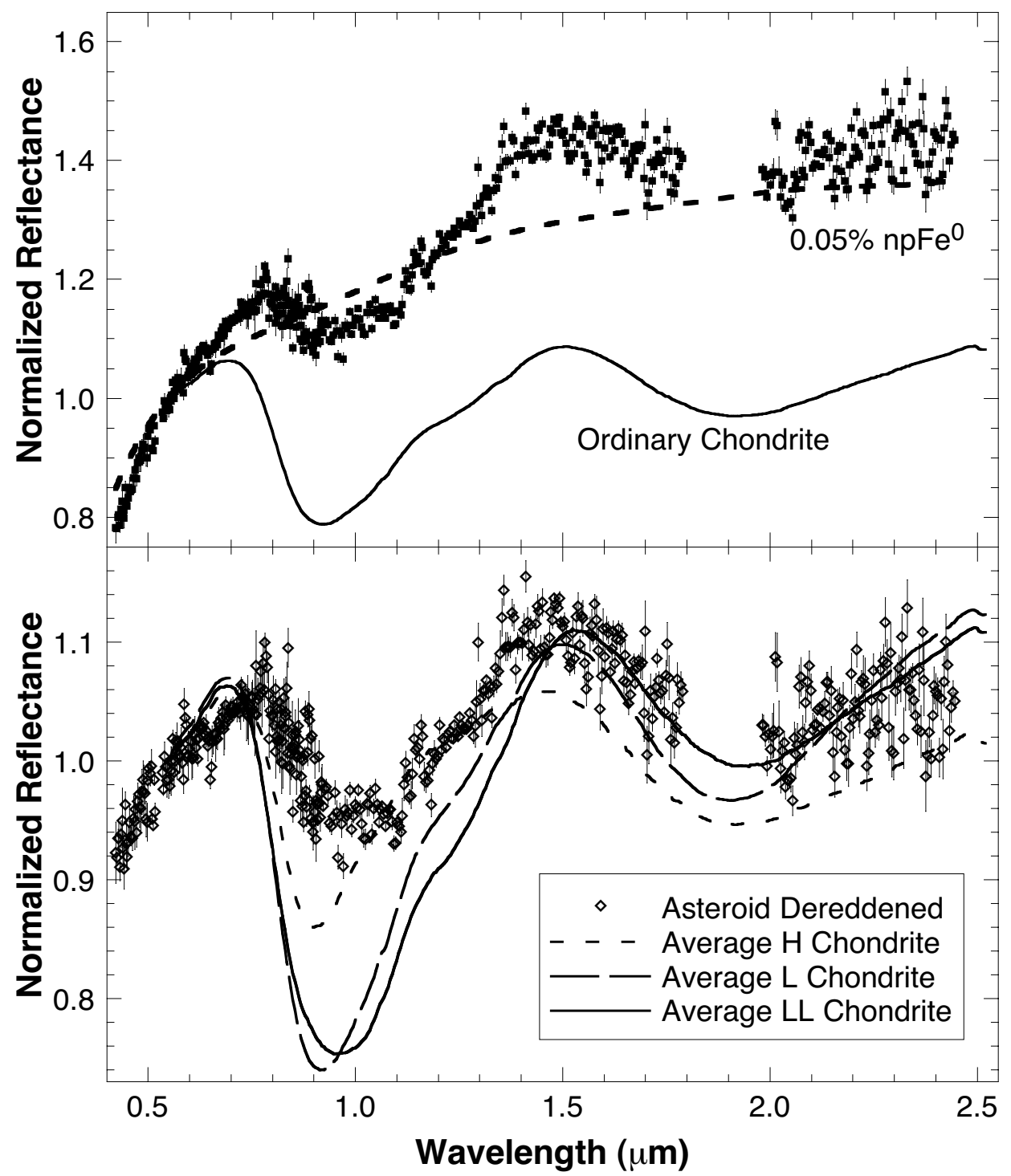

Fig. 5. Top panel: combined near-infrared plus visible spectrum of 4979 Otawara (data points), where the gap at $1.9 \mu \mathrm{m}$ is due to telluric water. For comparison, the dashed curve shows the reddening model for the presence of $0.05 \%$ nanophase iron $\left(\mathrm{npFe} \mathrm{e}^{0}\right.$ ) as determined by Pieters et al. (2001). The neutral slope of an ordinary chondrite meteorite (average LL chondrite) is shown for comparison. Bottom panel (with the vertical scale expanded to twice that of the top panel): Otawara spectrum (data points) de-reddened for $0.05 \%$ nanophase iron. Spectral slope and location of the $1 \mu \mathrm{m}$ and $2 \mu \mathrm{m}$ bands are consistent within the range of properties for ordinary chondrite meteorites (lines). All spectra are normalized to unity at $0.55 \mu \mathrm{m}$. Meteorite spectra are from Gaffey (1976).

primitive achondrites is greater than that for $\mathrm{S}(\mathrm{IV})$ asteroids (Burbine et al. 2001; Binzel et al. 2001a), we tried to make a comparison between Otawara and representative ordinary chondrites.

However, as has long been noted (see for example Wetherill \& Chapman 1988) and is evident in the upper panel of Fig. 5, spectra of S-class asteroids are significantly reddened compared with this meteorite analog, and this difference could probably be explained by space weathering effects. This process may be the result of dust impacts and solar wind sputtering on the surface of atmosphereless bodies and gives rise to a reddening of the spectral slope, a decrease of spectral absorption intensities and a diminishing of albedo. In fact surfaces of atmosphereless bodies exposed to the space environment for millions to billions of years are inevitably altered in physical, optical and/or chemical properties, as first demonstrated by analysis on the lunar soils (see for example Adams et al. 1971; Hapke et al. 1975).

While the effects of space weathering processes have long been debated, new results are supporting their validity. The Galileo mission for the first time demonstrated that a kind of alteration affects the surfaces of Gaspra, Ida and Dactyl (three S-type asteroids), modifying the reflectance spectra of fresh material to be redder, straighter and with shallower absorption bands, as reported by Chapman (1996). Recently the NEAR mission to 433 Eros (another S-type object) allowed a determination, thanks to X-ray and near-infrared spectrometer measurements, of an ordinary chondrite composition of Eros despite a red sloped, S-type spectrum, suggesting once again that some process such as reddening and/or darkening has altered the optical properties of the surface (Clark et al. 2000; Chapman 2000; McFadden et al. 2001; Bell et al. 2002). 
Recent work of Binzel et al. (2001b) shows also that there is a continuous transition in the spectral behaviour between a sample of small S-Q type Near Earth Objects and that of ordinary chondrites, arguing that a continuous natural process such as space weathering could affect these bodies in different ways, depending on their age and collisional history.

Moreover Pieters et al. (2000) demonstrated that the spectral properties of S-type asteroids are well modeled by the space weathering of ordinary chondrite material that is associated with accumulation of reduced nano-phase iron on soil grains. This kind of model was also successfully applied by Binzel et al. (2001a) for the interpretation of spectral differences between asteroid (25143) 1998 SF36, the S(IV) target of MUSES-C mission, and ordinary chondrites.

Following the de-reddening model developed by Binzel et al. (2001a), we divide the spectrum of Otawara by the curve for the minimal $(0.05 \%)$ component of nanophase iron. The results are shown in the lower panel of Fig. 5. We find an excellent match (within the noise of our spectrum) to the typical slopes for ordinary chondrite meteorites. Speculating on a more specific match, however, remains problematic. We find the $1 \mu \mathrm{m}$ band depth is shallow relative to the meteorite comparisons, but most consistent with the $\mathrm{H}$ chondrites. However, perhaps more diagnostic is the $1 \mu \mathrm{m}$ band center which is most consistent with LL chondrites, as is the depth of the $2 \mu \mathrm{m}$ band.

Thus, while we slightly favor an analogy between Otawara and LL chondrites, (based on the preceeding analysis and our OPX analysis), we consider this only a tentative result.

\section{Conclusion}

In conclusion, our results can be summarized as follows:

1. Our combined visible and infrared spectral data of Otawara put this object in the $\mathrm{S}$ taxonomic class composed of olivine and orthopyroxene.

2. Following the Gaffey et al. classification scheme (1993), Otawara belongs to the S(IV) subgroup.

3. A comparison between Otawara and H, L and LL ordinary chondrites shows the typical differences found in the literature between these meteorites and S-asteroids. Otawara could be a potential parent body of OC meteorites as the spectral reddening and shallow absorption features compared to OC spectra are consistent with trends that may be caused by space weathering processes. In fact a spectral analysis using current models for space weathering shows that the presence of only $0.05 \%$ nanophase iron may account for the spectral slope difference between Otawara and ordinary chondrite meteorites.

4. Our lightcurve data confirm the fast rotation period of Otawara $\left(P_{\text {syn }}=2.707 \pm 0.005 \mathrm{~h}\right)$ previously determined by other authors.

5. A first estimation of the complete spin state is presented with most likely a retrograde sense of rotation. Further observations during upcoming apparitions are needed to verify and improve this result.
All this information will allow the Rosetta scientists and mission planners to optimise the science operation planning during Otawara flyby, but only the analysis of the Rosetta data will permit a much more detailed investigation of the physical and compositional properties of 4979 Otawara.

Acknowledgements. The authors thank L. McFadden and P. Weissman for their detailed reviews and suggestions, and T. Cline who provided data for meteorite fields. Spin vector determination was performed after a suggestion by A. Erikson. T.M. was supported by the Polish KBN Grant 2 P03D 00718.

\section{References}

Adams, J. B., \& McCord, T. B. 1971, Proc. 2nd Lunar Sci. Conf., 2183

Bell, J. F., Izenberg, N. I., Lucey, P. G., et al. 2002, Icarus, 155, 119

Berthoud, M. G., Bell, J. F., Clark, B. E., \& Gaffey, M. J. 2001, Lunar Planet. Sci., 32, 2080

Binzel, R. P., Rivkin, A. S., Bus, S. J., Sunshine, J. M., \& Burbine, T. H. 2001a, Meteorit. Planet. Sci., 36, 116

Binzel, R. P., Harris, A. W., Bus, S. J., \& Burbine, T. H. 2001b, Icarus, 151,139

Bowell, E., Hapke, B., Domingue, D., et al. 1989, in Asteroids II (Univ. of Arizona Press, Tucson), 524

Burbine, T. H., McCoy, T. J., Nittler, L. R., \& Bell, J. F. 2001, Lunar Planet. Sci., 32, 1860

Bus, S. J., Ph.D. Thesis, Massachusset Institute of technology, Cambridge, MA, USA

Chapman, C. R. 1996, Meteoritics \& Plan. Sci., 31, 699

Chapman, C. R., \& Near MSI-Nis Team 2000, Meteoritics \& Plan. Sci., 35,39

Clark, B. E., Bell, J. F., Veverka, J., et al. 2000, Meteoritics \& Plan. Sci., 35, 41

Cloutis, E. A., Gaffey, M. J., Jackowski, T. L., \& Reed, K. L. 1986, J. Geoph. Res., 91, 11641

Doressoundiram, A., Weissman, P. R., Fulchignoni, M., et al. 1999, A\&A, 352, 697

Gaffey, M. J. 1976, J. Geoph. Res., 81, 905

Gaffey, M. J., Burbine, T. H., Piatek, J. L., et al. 1993, Icarus, 106, 573

Gaffey, M. J. 1999, Lunar Planet. Sci., 30, 1375

Gaffey, M. J. 2000, Lunar Planet. Sci., 31, 1092

Gaffey, M. J. 2001, Lunar Planet. Sci., 32, 1587

Hapke, B., Cassidy, W., \& Wells, E. 1975, The moon, 13, 339

Harris, A. W., Young, J. W., Bowell, E., et al. 1989, Icarus, 77, 171

Jarosewich, E. 1990, Meteoritics, 25, 323

Le Bras, A., Dotto, E., Fulchignoni, M., et al. 2001, A\&A, 379, 660

Michałowski, T. 1993, Icarus, 106, 563

McFadden, L. A., Wellnitz, D. D., Schnaubelt, M., et al. 2001, Meteoritics \& Plan. Sci., 36, 1711

McSween, H. Y., Bennett, M. E., \& Jarosewich, E. 1991, Icarus, 90, 107

Pieters, C. M., Taylor, L. A., Noble, S. K., et al. 2000, Meteoritics \& Plan. Sci., 35, 1101

Rayner, J. T., Toomey, D. W., Onaka, P. M., et al. 1998, Proc. SPIE, 3354, 468-479

Wetherill, G. W., \& Chapman, C. R. 1988, Asteroids and meteorites, in Meteorites and the Early Solar System, ed. J. F. Kerridge, \& M. S. Matthews (University of Arizona Press, Tucson, Arizona), 35

Xu, S., Binzel, R. P., Burbine, T. M., \& Bus, S. J. 1995, Icarus, 115, 1 Zappala, V., Cellino, A., Barucci, M. A., et al. 1990, A\&A, 231, 548 